medgen $2019 \cdot 31: 364-371$

https://doi.org/10.1007/s11825-019-00271-6

Online publiziert: 12. Dezember 2019

(c) Der/die Autor(en) 2019

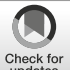

\author{
Nico Maximilian Jandl ${ }^{1,2} \cdot$ Alexander Volk $^{3}$. Florian Barvencik ${ }^{1}$ \\ ${ }^{1}$ Institut für Osteologie und Biomechanik (IOBM), Universitätsklinikum Hamburg-Eppendorf, Hamburg, \\ Deutschland \\ ${ }^{2}$ Klinik und Poliklinik für Orthopädie, Universitätsklinikum Hamburg-Eppendorf, Hamburg, Deutschland \\ ${ }^{3}$ Institut für Humangenetik, Universitätsklinikum Hamburg-Eppendorf, Hamburg, Deutschland
}

\title{
Hypophosphatasie - eine klinisch und genetisch variable Erkrankung
}

meist tödlich war. Durch Störungen im Vitamin-B6-Stoffwechsel kann es zu epileptischen Anfällen kommen [3].

Davon abzugrenzen ist die pränatale benigne HPP, bei welcher der fetale Ultraschall zwar kürzere und gebogene lange Röhrenknochen zeigt, aber keine Frakturen sichtbar sind und die körperlichen Untersuchungsbefunde im dritten Trimester rückläufig sind. Nach der Geburt kann das Spektrum der pränatal benignen HPP von klinisch asymptomatisch bis hin zu Symptomen einer infantilen Form der HPP reichen, die dann innerhalb der ersten sechs Monate nach Geburt auftreten.

Erste klinische Anzeichen der infantilen HPP können Schwäche, unzureichende Nahrungsaufnahme, Wachstumsstörungen und Thorax- und Schädeldeformitäten durch eine Kraniosynostose sein. Weitere Symptome umfassen Frakturen und metaphysäre rachitische Knochenveränderungen. Eine Hypercalcämie mit vermehrter Calciumexkretion kann zur Nephrocalcinose führen. Eine verringerte Körpergröße im Erwachsenenalter kommt häufig vor [1].

Die kindliche Form überlappt in ihrem klinischen Spektrum mit der Säuglings- und der Erwachsenenform und ist definiert durch ein Auftreten von Symptomen zwischen 6 Monaten und 18 Jahren. Sie ist durch einen verfrühten Verlust der Milchzähne samt intakter Wurzel vor dem 5. Lebensjahr, Zahn-/Kieferfehlstellungen, Skelettdeformitäten wie gebogene Röhrenknochen und vergrößerte Gelenke, sowie
Muskelschwäche und selten Kleinwuchs gekennzeichnet $[1,4]$.

Die spätmanifeste adulte Form ist in ihrer Ausprägung besonders heterogen, insgesamt jedoch mit milderen Symptomen verbunden als bei kindlichen Formen. Im mittleren Lebensalter kann es gehäuft zu Stressfrakturen, Knochenmarködemen, Frakturheilungsstörungen, Osteomalazie und Zahnproblemen kommen (• Abb. 1; [1]). In vielen Fällen zeigt sich diese Form lediglich durch unspezifische Symptome wie Muskelund Knochenschmerzen, Muskelschwäche, Fatigue, Verkalkungen von Sehnen oder Gelenken, Migräne und Depressionen [5, 6]. Die hohe Prävalenz dieser unspezifischen Symptome in der Allgemeinbevölkerung erschwert jedoch oftmals die eindeutige Zuordnung der Symptome zur HPP, auch wenn charakteristische Laborveränderungen und ALPL-Genmutationen bei betroffenen Patienten nachweisbar sind.

Die Odontohypophosphatasie stellt eine Sonderform der HPP dar und manifestiert sich ausschließlich an den Zähnen. Sie ist die mildeste und wahrscheinlich verbreitetste Form der HPP [7] und charakterisiert durch einen frühen Verlust von Milchzähnen und/oder bleibenden Zähnen. Mineralisationsstörungen der Zähne können zu einem starken Kariesbefall führen und den Zahnhalteapparat schwächen [3]. 


\begin{tabular}{|c|c|c|c|}
\hline HPP-Unterform & Erstmanifestation & Vererbung & Klinische Charakteristika \\
\hline Perinatal & In utero & $A R>A D$ & $\begin{array}{l}\text { Thoraxdeformität, Lungenhypoplasie, Frakturen, Skeletthypomineralisation, gebogene } \\
\text { und verkürzte Röhrenknochen, epileptische Anfälle, Hypercalcämie }\end{array}$ \\
\hline Pränatal benigne & In utero & $A R<A D$ & $\begin{array}{l}\text { Gebogene Röhrenknochen ohne Frakturen in utero, Rückbildung ab 3. Trimester, } \\
\text { im Verlauf Spektrum von infantiler bis Odontohypophosphatasie }\end{array}$ \\
\hline Infantil & $<6$ Monate & $A R>A D$ & $\begin{array}{l}\text { Rachitiszeichen (Thorax, Metaphysen der Röhrenknochen), Kraniosynostose, verringerte } \\
\text { Körpergröße im Erwachsenenalter, epileptische Anfälle, Hypercalcämie, Nephrocalcinose, } \\
\text { frühzeitiger Milchzahnverlust }\end{array}$ \\
\hline Kindlich & $\begin{array}{l}6 \text { Monate- } \\
18 \text { Jahre }\end{array}$ & $A R>A D$ & $\begin{array}{l}\text { Rachitiszeichen, vergrößerte Gelenke, gebogene Röhrenknochen, Kraniosynostose, } \\
\text { chronische Knochenmarködeme, Muskel-, Gelenk- und Knochenschmerzen, } \\
\text { Muskelschwäche, frühzeitiger (Milch-)Zahnverlust }\end{array}$ \\
\hline Adult & $>18$ Jahre & $A R \approx A D$ & $\begin{array}{l}\text { Stressfrakturen, Frakturheilungsstörungen, Chondrocalcinose, Tendinosis calcarea, } \\
\text { spinale Hyperostose, Muskelschwäche, Muskelschmerzen, Gelenkschmerzen, } \\
\text { Osteomalazie, frühzeitiger (Milch-)Zahnverlust, Migräne, Depressionen }\end{array}$ \\
\hline Odonto & $>6$ Monate & $A R<A D$ & Frühzeitiger (Milch-)Zahnverlust, schwere Karies, keine Skelettmanifestation \\
\hline
\end{tabular}

\section{Klinische Diagnostik}

Die fehlende bzw. reduzierte Aktivität der AP führt zu einer Erhöhung verschiedener Substrate im Serum bzw. Urin, z. B. von anorganischem Pyrophosphat, Pyridoxal-5-Phosphat (PLP) und Phosphoethanolamin. Zur klinischen Diagnostik der HPP gehört daher neben der Bestimmung der AP und knochenspezifischen AP auch die Bestimmung des PLP aus dem Serum, welches als das aussagekräftigste der drei Substrate angesehen wird. Generell ist eine erniedrigte Aktivität der AP im Serum der entscheidende Hinweis auf das Vorliegen einer ALPL-Genmutation [8]. Patienten mit schwerer HPP zeigen im Allgemeinen eine geringere altersspezifische AP-Aktivität, während der Schweregrad einer adulten HPP besser mit dem PLP-Spiegel korreliert [5, 9]. Zur korrekten Interpretation ist es wichtig, dass Patienten mind. zwei Wochen vor dem Zeitpunkt der Blutabnahme keine Nahrungsergänzungsmittel mit Vitamin B6 zu sich genommen haben, da es hierunter $\mathrm{zu}$ falsch-erhöhten PLP-Spiegeln kommen kann. Bei der Abnahme von PLP ist auf einen lichtgeschützten und eisgekühlten Transport zu achten. Die AP und die knochenspezifische AP sind im Vergleich $\mathrm{zu}$ alters- und geschlechtsspezifischen Referenzwerten einzuordnen. Andere Ursachen einer AP-Erniedrigung (Hypophosphatasämie, - Tab. 2) und Differentialdiagnosen der HPP (• Tab. 3) sollten klinisch oder laborchemisch aus- geschlossen werden. Hierzu zählen u.a. die Medikation mit Bisphosphonaten, Denosumab oder Glucocorticoiden, als auch andere Erkrankungen wie adyname Knochenerkrankung, multiples Myelom, Osteogenesis imperfecta, Hypothyreose, Zöliakie, Vitamin-D-Intoxikation oder Milch-Alkali-Syndrom [5].

\section{Pathophysiologie}

Die gewebsunspezifische AP gehört zur Gruppe der alkalischen Phosphatasen und wird im Gegensatz zu den gewebsspezifischen AP (intestinale AP, plazentare AP und plazentaähnliche AP) ubiquitär und stark in Leber, Niere und Knochen exprimiert. Im Gewebe ist die gewebsunspezifische AP über Glycosylphosphatidinositol (GPI) membrangebunden [10]. Im Blut ist die gewebsunspezifische AP löslich und lipidungebunden [11]. Für die katalytische Aktivität ist eine Homodimerisierung zweier AP-Monomere notwendig, was auch die allosterischen Eigenschaften des Enzyms erklärt [12]. Verschiedene pathophysiologische Theorien zu den zu beobachteten klinischen Befunden bestehen: Die zentrale Rolle bei der Knochenmineralisierung erklärt sich z.B. durch die Funktion der gewebeunspezifischen AP als Hydrolase von Pyrophosphat. Entstehendes anorganisches Phosphat bildet zusammen mit Calcium Hydroxylapatitkristalle, den Hauptbestandteil der Knochenmatrix (• Abb. 2). Im Nervengewebe hydroly- siert die gewebsunspezifische AP u.a. PLP, das phosphorylierte Vitamin B6. Das unphosphorylierte Vitamin B6 kann die Blut-Hirn-Schranke überwinden, rephosphoryliert werden und als Koenzym u. a. bei der Synthese des Neurotransmitters GABA wirken. Weiterhin hat die gewebsunspezifische AP eine Rolle bei der Hirnentwicklung und insbesondere bei der Reifung neuronaler Stammzellen, der Synapsenbildung und Myelinisierung, was mit Symptomen wie Migräne, Schmerzzuständen, Angststörungen und Depressionen bei HPPPatienten in Verbindung gebracht wird [6].

\section{Genetische Diagnostik}

Perinatale und frühkindliche Formen sind meist autosomal-rezessiv vererbt, während die benigne pränatale, die im Kindesalter beginnende, die adulte HPP und die Odontohypophosphatasie autosomal-rezessiv oder autosomaldominant vererbt werden.

Prinzipiell handelt es sich bei der HPP um eine klinische Diagnose, die aufgrund klinischer, laborchemischer und radiologischer Befunde gestellt wird. Eine molekulargenetische Untersuchung des $A L P L$-Gens ist für die Diagnose nicht zwingend erforderlich, wird aber zur differentialdiagnostischen Abgrenzung und zur Einschätzung von Wiederholungswahrscheinlichkeiten bei zukünftigen Schwangerschaften angeboten und ist 
medgen 2019·31:364-371 https://doi.org/10.1007/s11825-019-00271-6

(c) Der/die Autor(en) 2019

\section{N. M. Jandl · A. Volk · F. Barvencik}

\section{Hypophosphatasie - eine klinisch und genetisch variable Erkrankung}

\section{Zusammenfassung}

Die Hypophosphatasie (HPP) ist eine erbliche metabolische Multisystemerkrankung, deren klinische Hauptcharakteristika Mineralisierungsstörungen von Knochen und Zähnen sowie Muskel- und Gelenkschmerzen sind. Die klinische Symptomatik ist vom Erkrankungsalter abhängig und gestaltet sich sowohl interindividuell als auch intrafamiliär sehr variabel. Es werden sechs Unterformen der HPP abgegrenzt, wobei die Übergänge fließend sind. Sie reichen von der schweren perinatalen Form, die früher aufgrund fehlender Skelettmineralisierung meist tödlich war, bis hin zur adulten Form mit typischen Symptomen wie Frakturheilungsstörungen oder Stressfrakturen. Unspezifische Symptome wie Muskelschmerzen und -schwäche,
Migräne oder Depressionen können ebenfalls Teil der HPP sein. Während schwere Formen mit einer Prävalenz zwischen 1/100.000 und $1 / 300.000$ selten sind, kommen milde Formen der HPP deutlich häufiger vor. Perinatale und frühkindliche Formen sind meist autosomalrezessiv vererbt, hingegen werden später auftretende Formen autosomal-rezessiv oder -dominant vererbt. Ursache der HPP ist eine reduzierte oder fehlende Aktivität der gewebeunspezifischen alkalischen Phosphatase (AP), welche durch das ALPL-Gen kodiert wird. Laborchemisch lassen sich im Serum eine alters- und geschlechtsspezifisch erniedrigte AP-Aktivität und eine konsekutive Erhöhung der AP-Substrate, z. B. des Pyridoxal5-Phosphats (PLP), feststellen. Seit der
Erstbeschreibung der Erkrankung 1948 haben sich die Diagnostik und Therapie der HPP dramatisch verbessert. Vor 4 Jahren ist eine Enzymersatztherapie mit Asfotase alfa (Strensiq ${ }^{\circledR}$ ) für schwer betroffene HPPPatienten mit Beginn der Erkrankung vor dem 18. Lebensjahr zugelassen worden. Dieser Artikel gibt einen Überblick über das breite klinische Spektrum der HPP, pathophysiologische Hintergründe, die laborchemische und molekulargenetische Diagnostik sowie gegenwärtige Therapieoptionen und deren Behandlungsindikationen.

\section{Schlüsselwörter}

HPP · Genetik · ALPL-Gen · Asfotase alfa . Strensiq

\section{Hypophosphatasia: a clinically and genetically variable disease}

\begin{abstract}
Hypophosphatasia (HPP) is a hereditary metabolic multisystemic disease whose main clinical characteristics are mineralization disorders of bones and teeth as well as muscle and joint pain. The clinical symptoms depend on the age of onset of HPP and are highly variable, both interindividually and within families. Six subtypes of HPP are defined, with fluent transitions. They range from the severe perinatal form, which was previously lethal owing to a lack of skeletal mineralization, to the adult form, with typical symptoms such as fracture healing disorders or stress fractures. Nonspecific symptoms, such as muscle pain and weakness, migraine or depression, can also be part of HPP. Although
\end{abstract}

severe forms, with a prevalence between $1 / 100,000$ and $1 / 300,000$, are rare, mild forms of HPP are much more common. Perinatal and manifestations in early childhood are mostly inherited in an autosomal-recessive manner, whereas those with later onset are inherited in an autosomal-recessive or autosomaldominant manner. HPP is caused by reduced or absent activity of the tissue-nonspecific alkaline phosphatase (AP), which is encoded by the ALPL gene. Laboratory testing of serum shows an age- and gender-specific low AP activity and a consecutive increase in AP substrates, e.g., pyridoxal-5-phosphate (PLP). Since the disease was first described in 1948, the diagnosis and treatment of HPP have improved dramatically. Four years ago, enzyme replacement therapy with asfotase alfa (Strensiq ${ }^{\circledR}$ ) was approved for severely affected HPP patients with the onset of the disease before the age of 18 years. This article provides an overview of the broad clinical spectrum of HPP, pathophysiological background, laboratory and molecular genetic diagnostics, as well as current therapy options and their treatment indications.

Keywords

HPP - Genetics · ALPL gene - Asfotase alfa . Strensiq bei Inanspruchnahme vorgeburtlicher Untersuchungen notwendig $[1,13]$.

Derzeit sind knapp 400 verschiedene Krankheitsallele des ALPL-Gens beschrieben. In der überwiegenden Mehrzahl handelt es sich um Missense-Mutationen $(\sim 70 \%)$ gefolgt von kleineren Deletionen ( $12 \%)$, Splicesite- $(\sim 6 \%)$ sowie Nonsense-Mutationen ( 4\%) bzw. kleineren Insertionen $(\sim 4 \%)$. Größere Deletionen sind insgesamt seltener $(\sim 3 \%)$, wobei eine routinediagnostisch einfach anwendbare, kommerzielle Nachweismethode (SALSA ${ }^{\circledR}$ MLPA $^{\circledR}$ Probemix) erst seit Ende 2018 zur Verfügung steht, was einen Bias bei älteren Publikationen hervorrufen könnte. Einen Hotspot von Krankheitsallelen innerhalb des $A L P L$ Gens gibt es nicht [14] und Neumutationen sind selten [15]. Zwei Fälle einer HPP infolge einer uniparentalen Isodisomie sind beschrieben [16, 17]. Ein Founder-Effekt ist für spezifische Mutationen in manchen Populationen beschrieben [18].

\section{Genotyp-Phänotyp-Korrelation und genetische Beratung}

In Abhängigkeit des jeweiligen Krankheitsallels wurde bei Überexpressionsanalysen eine Variabilität der enzymatischen Restaktivität festgestellt, die auch eine Korrelation zu den klinischen Befunden erlaubte [19]. In kleineren Kollektiven mit Patienten mit rekurrenten Genotypen (50 bzw. 105 Patienten) waren jeweils ähnliche klinische Befunde bei gleichem Genotyp beschrieben worden $[1,7]$. Jedoch ist eine intrafamiliäre 


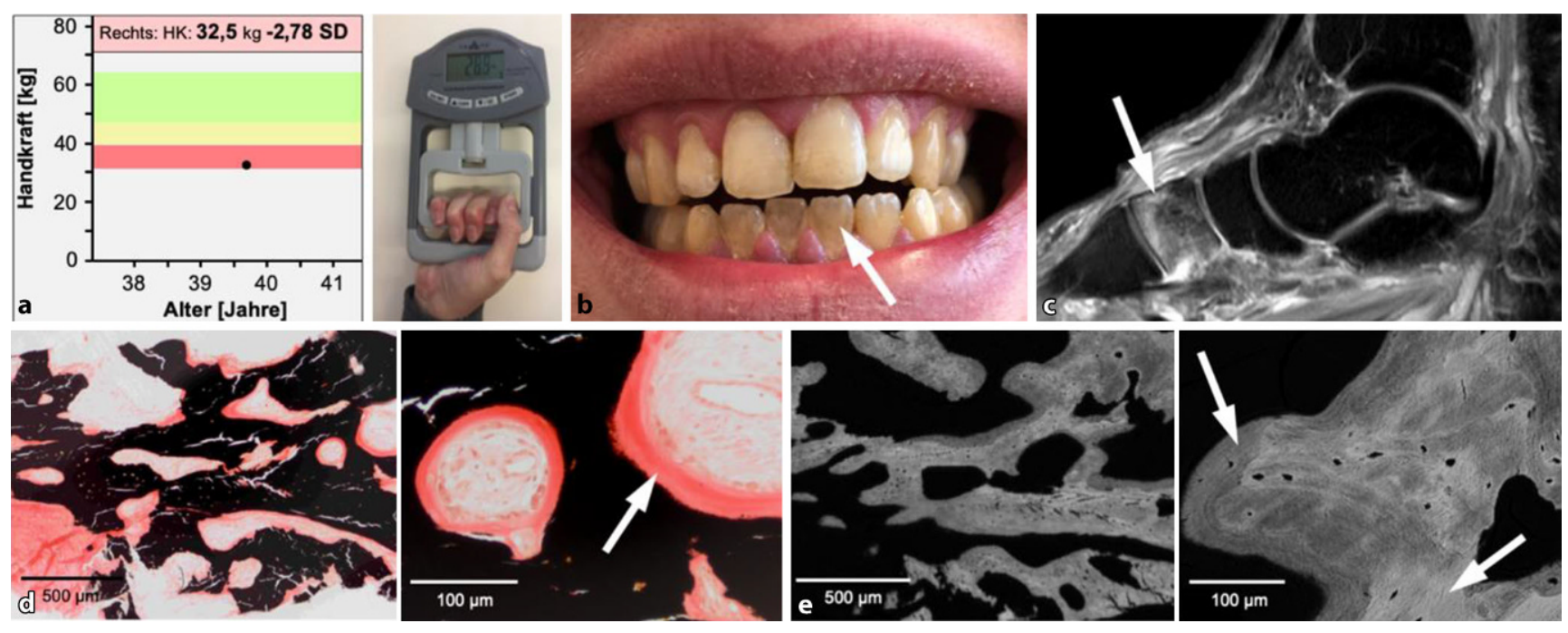

Abb. 1 ॥ Klinische und histologische Charakteristika der Hypophosphatasie (HPP).

a Die reduzierte Muskelkraft lässt sich mithilfe eines Handdynamometers objektivieren. Im Beispiel zeigt der Patient eine im Vergleich zu alters- und geschlechtsspezifischen Referenzwerten um fast 3 Standardabweichungen (SD) verringerte Handkraft (HK) rechts.

b Typische Zahnveränderungen mit durch die Hypomineralisation bedingter Verfärbung der Unterkieferschneidezähne bei einem Patienten mit adulter Hypophosphatasie.

c Rezidivierende Knochenmarködeme oder Stressfrakturen des Fußskeletts stellen häufige Symptome einer adulten HPP dar. Der Pfeil kennzeichnet ein Knochenmarködem im Os cuneiforme mediale im MRT.

d Histologischer Schnitt einer Knochenbiopsie des Os pubis in Von-Kossa-Färbung. Schwarz gefärbt kommt mineralisierter Knochen zur Darstellung. Auffällig sind die zahlreichen, rosafarbenen, aufgelagerten, dicken Schichten von nicht mineralisiertem Knochen (Osteoid) im Sinne einer Osteomalazie bei adulter HPP.

e Korrespondierender Schnitt unter dem Rasterelektronenmikroskop zur Beurteilung des Mineralisationsgrades. Im mineralisierten Knochen finden sich große Anteile mit niedriger Mineralisation (dunkelgrau) neben Arealen mit normaler Mineralisation (hellgrau).

Variabilität nicht nur bei heterozygoten Mutationsträgern, sondern auch innerhalb von Familien mit autosomal-rezessiver HPP zu beobachten [20, 21]. Auch entgegen der o.g. Studien scheint die Variabilität bei gleichem Genotyp größer zu sein [22]. Eine klare Abgrenzung von autosomal-dominanter und autosomalrezessiver Vererbung erscheint nicht immer möglich, da innerhalb einer Familie heterozygote Mutationsträger mit mildem klinischen Phänotyp („dominant erblich") neben Patienten mit biallelischen Mutationen und ausgeprägter klinischer Symptomatik (,rezessiv erblich") auftreten können [1]. Insgesamt erscheint aktuell eine Prognose zum klinischen Phänotyp anhand eines Genotyps nicht möglich. Inwieweit z. B. ein quadriallelisches Modell [23] zukünftig zur Erklärung der phänotypischen Variabilität beitragen kann oder ob genetische Modifier identifiziert werden, bleibt abzuwarten.

Eine besondere Herausforderung für die genetische Beratung und Diagnos- tik stellen vorgeburtliche Untersuchungen und dabei die Abgrenzung der perinatal schweren von der pränatal benignen HPP dar. Historisch gesehen war eine perinatale Manifestation zusammen mit einem Mutationsnachweis in beiden ALPLGenkopien mit einer infausten Prognose verbunden (nachgeburtlich letal), jedoch sind auch Patienten mit benigner pränataler HPP und biallelischen Mutationen beschrieben: Bei 1/3 aller Patienten mit beniger pränataler HPP werden biallelische ALPL-Mutationen nachgewiesen; in $2 / 3$ der Patienten mit benigner pränataler HPP liegt eine heterozygote $A L P L$ Mutation vor [24].

\section{Therapie der Hypophosphatasie - ein multidisziplinärer Therapieansatz}

Die Therapie der HPP erfordert einen multidisziplinären Ansatz und eine gute Teamarbeit in einem spezialisierten Zentrum. Dabei sind für eine umfassende Betreuung von HPP-Patienten Vertreter der
Disziplinen Pädiatrie, Osteologie, Orthopädie, Ophthalmologie, Traumatologie, Labormedizin, Humangenetik, Radiologie, Neurologie, Neurochirurgie, Zahnmedizin, Kieferorthopädie, Ernährungsmedizin, Psychologie und Physiotherapie von zentraler Bedeutung. In Abhängigkeit des Alters der Patienten und der Ausprägung der HPP-Erkrankung wird die Nutzung der personellen und apparativen Ressourcen von einem spezialisierten Zentrum für den individuellen Fall koordiniert und in Diagnostik und Therapie gezielt eingesetzt.

\section{Supportive Therapie}

Die nicht medikamentöse Therapie der HPP umfasst neben allgemein orthopädischen Maßnahmen bei Frakturen, Stressfrakturen, Knochenödemen oder Frakturheilungsstörungen physiotherapeutische Anwendung zum Erhalt und zur Verbesserung der Funktion des Bewegungsapparates sowie eine HPPspezifische Ernährungsberatung. Auch 
Tab. 2 Andere Ursachen für eine Erniedrigung der alkalischen Phosphatase (AP) Ursachen einer AP-Erniedrigung

Achondroplasie

Antiresorptiva (Bisphosphonate, Denosumab)

Chemotherapie

CKD-MBD, adyname Knochenerkrankung

Cushing-Syndrom

Große Operationen/Traumata

Hypoparathyreoidismus

Hypothyreoidismus

Kleidokraniale Dysplasie

Massive Bluttransfusionen

Milch-Alkali-Syndrom

Morbus Wilson

Multiples Myelom

Osteogenesis imperfecta

Schwere (perniziöse) Anämie

Unterernährung

Vitamin-D-Intoxikation

Zink-, Magnesium-, Vitamin-C-, Vitamin-B6-, Vitamin-B12-, Folsäure-Mange

Zöliakie

CKD-MBD Chronic Kidney Disease - Mineral Bone Disorder

die zahnärztlich präventiven Maßnahmen und regelmäßige Mundhygiene sind ebenso wichtig zum bestmöglichen Erhalt des Kauapparates wie die zahnärztlich-kieferorthopädische Therapie bei Zahnverlust und/oder Zahnfehlstellung.

Es sollte eine optimale, an die HPP angepasste Einstellung des Knochenstoffwechsels erfolgen. Ziel ist hier die Vermeidung von Mangelzuständen von wichtigen nutritiven Faktoren für das Skelettsystem (z. B. Vitamin D, Folsäure, Vitamin B 12, Magnesium, Zink und andere). Dabei ist jedoch zu berücksichtigen, dass z. B. bei der Vitamin D Dosierung die besondere Pathophysiologie der HPP berücksichtigt und der Vitamin-D-Spiegel nicht zu stark angehoben wird, um eine erhöhte Phosphatrückresorption zu vermeiden.

\section{Medikamentöse Therapie bei HPP}

Die medikamentöse Therapie der HPP dient zum einen der gezielten Analgesie und der Suppression der entzündli-

\begin{tabular}{l} 
Tab. 3 Differentialdiagnosen der \\
Hypophosphatasie. \\
\hline Differentialdiagnose \\
\hline Chondrodysplasien \\
Cole-Carpenter-Syndrom \\
Dentinogenesis imperfecta \\
Idiopathische juvenile Osteoporose \\
Kampomele Dysplasie \\
Kleidokraniale Dysplasie \\
Osteogenesis imperfecta \\
Osteomalazie
\end{tabular}

chen Aktivität im Organismus. Hier wurde durch Whyte et al. gezeigt, dass z.B. Naproxen den Schmerz bei Knochenmarködemen lindern kann und zur Reduktion des Entzündungsstatus beitragen kann [25]. Auch die Schmerzen durch Hydroxyapatit und/oder Calciumpyrophosphatkristallablagerungen in Gelenken scheinen auf die Gabe nichtsteroidaler antiinflammatorischer Medikamente anzusprechen $[26,27]$. Zum anderen sind die Verbesserung der Knochenqualität und die Therapie der osteomalazischen Komponente der HPP ein wesentliches Ziel der medikamentösen Therapie. Hier sind in der Vergangenheit Versuche unternommen worden, mit löslicher alkalischer Phosphatase bei Patienten mit Morbus-Paget-Erkrankung oder humaner plazentarer alkalischer Phosphatase die defekte AP von HPP-Patienten zu ersetzen [28]. Dabei konnte zwar ein passagerer Anstieg der AP im Serum erzielt werden, jedoch keine klinische oder radiologische Verbesserung der HPP-Befunde. Antiresorptive Medikamente wie z. B. Bisphosphonate sind kontraindiziert bei der HPP, da sie als PPI-Analoga bei HPP direkt die Mineralisation hemmen oder durch die Bindung von Zink oder Magnesium die Restfunktion der AP weiter supprimieren können. Im Gegensatz zu den antiresorptiven Medikamenten wurden in Einzelfällen für osteoanabole Medikamente (z.B. Teriparatid), welche die Aktivität der AP erhöhen, teilweise positive Effekte wie Frakturheilung oder Suppression von Knochenmarködemen gezeigt [29]. Weiterhin wurde in einer Open Label Phase 2 A Single-Center-Studie als osteoanaboles Medikament ein Sclerostin-Antikörper (BPS804) ver- wendet, der bei HPP-Patienten zu einem Anstieg der Knochenformationsparameter und zu einer Verbesserung der Knochendichte geführt hat [30].

\section{Enzymersatztherapie bei HPP}

Eine gezielte Enzymersatztherapie steht seit 4 Jahren zur Verfügung und stellt nun eine relevante medikamentöse Option für den von der HPP schwer betroffenen Patienten dar. Asfotase alfa (AA) ist ein „First-in-class“-Medikament, welches über einen Deca-Aspartat-Anker an das Hydroxyapatit der mineralisierten Knochenoberfläche anhaftet und mit durch das Fc-Fragment von IgG1 verlängerter Halbwertzeit die katalytische Aktivität der AP übernimmt [31]. Es ist ein humanes rekombinantes, gewebeunspezifisches alkalisches PhosphataseFc-Deca-Aspartat-Fusionsprotein, das zwei identische Polypeptidketten mit einer Länge von jeweils 726 Aminosäuren umfasst und aus der katalytischen Domäne der humanen gewebeunspezifischen AP, der humanen Immunglobulin-G1Fc-Domäne und einer Deca-AspartatPeptid-Domäne besteht [32].

In der ersten klinischen Open-LabelPhase 2-Studie erhielten 11 schwer betroffene Patienten mit perinataler und infantiler HPP Asfotase alfa. Die Auswertung der 9 Patienten, die die Studie abgeschlossen haben, zeigte, dass es zu einer deutlichen Verbesserung der Knochenmineralisation, der Muskelkraft und der Atemfunktion gekommen war [31]. Die Analyse der 7-JahresErgebnisse der oben genannten Studie bestätigt eine prolongierte Verbesserung der skelettalen Mineralisation, der respiratorischen Funktion, des Wachstums und der motorischen Entwicklung bei insgesamt guter Verträglichkeit [33]. Mittlerweile wurden auch Studienergebnisse veröffentlicht, die eine Wirksamkeit bei erwachsenen HPP-Patienten zeigen. Kishnani et al. haben in einer Studie mit 19 erwachsenen HPP-Patienten, bei der 14 HPP-Patienten die Studie abgeschlossen haben, gezeigt, dass eine signifikante Verbesserung des 6-min-Walk-Tests (6MWT) und eine verbesserte Mineralisationszeit des Knochens erzielt werden konnte [4]. In einer Multiscale-Analyse 


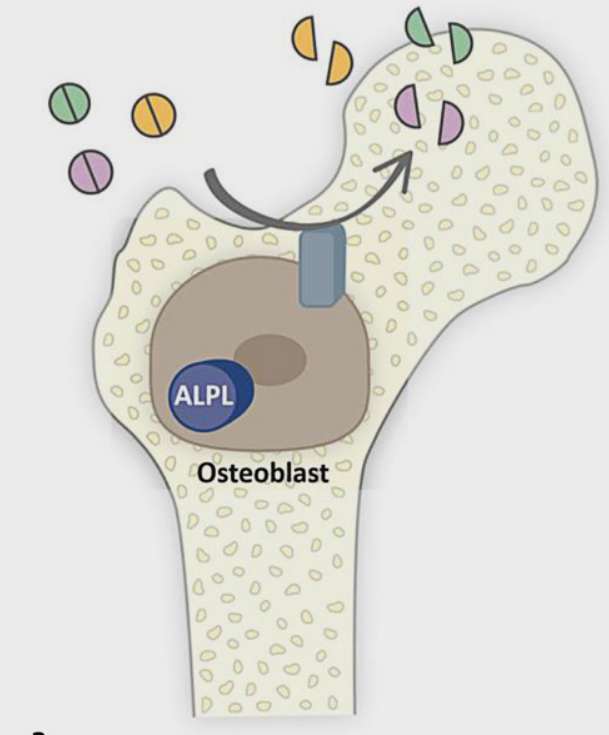

a

PPi (Pyrophosphat)
PLP (Pyridoxal-5-Phosphat)

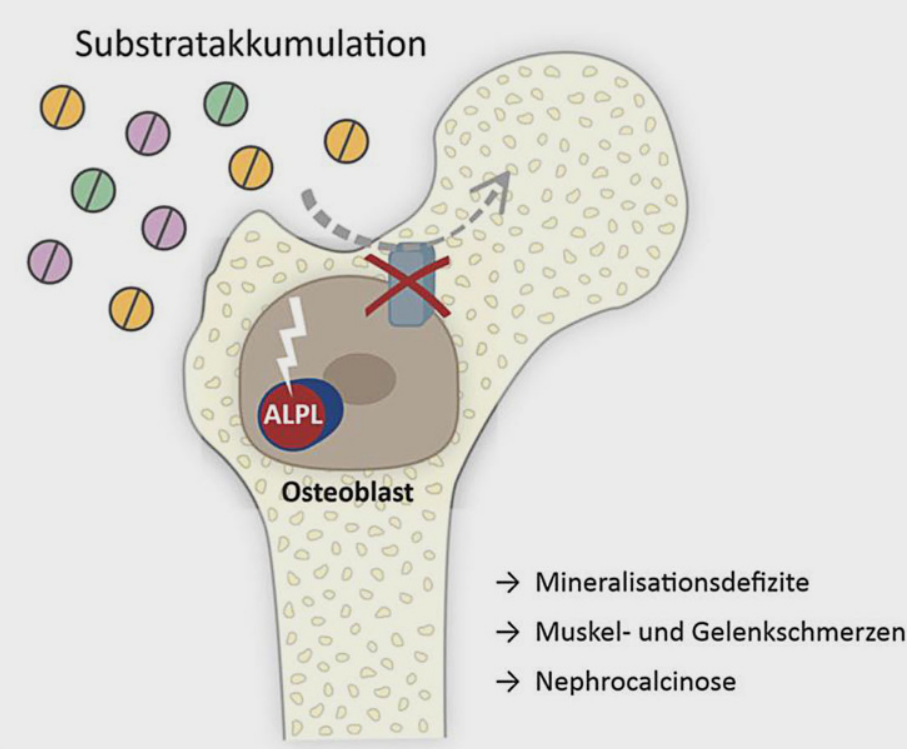

PEA (Phosphoethanolamin)
AP (Alkalische Phosphatase)

Abb. 2 A Schematische Darstellung der pathophysiologischen Vorgänge bei Hypophosphatasie (HPP). a Normal. b HPP.

der Knochenqualität unter Enzymersatztherapie wurde in einer Fallbeobachtung darüber hinaus histologisch eine Verbesserung der Knochenmineralisation sowie anderer Knochenqualitätsparameter beobachtet [34]. Hinzu kommen Einzelfallberichte, dass auch nach jahrelang bestehenden Pseudarthrosen z. B. des Femurs bei einliegendem Marknagel noch die Ausheilung der Pseudarthrose unter Enzymersatztherapie erzielt werden kann [35]. In Deutschland ist die Enzymersatztherapie zugelassen für die Langzeit-Enzymersatztherapie bei Patienten, bei denen die Hypophosphatasie im Kindes- und Jugendalter aufgetreten ist, um die Knochenmanifestationen der Krankheit zu behandeln.

Vor dem Hintergrund der Seltenheit der Erkrankung und der dadurch begrenzten Patientenzahl, die in Studien eine Enzymersatztherapie erhalten haben, und unter Berücksichtigung des Nebenwirkungsprofils (Injektionsreaktionen der Haut und des subkutanen Fettgewebes, allergische Reaktionen u.a.) ist die Indikationsstellung im individuellen Fall sehr genau zu prüfen. Es ist eine sorgfältige Abwägung des klinischen Nutzens und der Risiken sowie der bisher fehlenden Erfahrung bei Lang- zeitanwendung vorzunehmen. Shapiro und Lewiecki empfehlen aufgrund der begrenzten Erfahrung, der Kosten der Therapie und der vor allem bei erwachsenen HPP-Patienten überschaubaren Datenlage, die Anwendung nur bei sehr schwer betroffenen Patienten mit gut dokumentierter Krankengeschichte in Erwägung zu ziehen [36].

Insgesamt haben sich die therapeutischen Optionen nicht zuletzt und vor allem durch die Enzymersatztherapie suffizient erweitert, sodass die Versorgung von Patienten, die von einer Hypophosphatasie betroffen sind, besser ist als noch vor einigen Jahren. Dennoch ist klinisch und wissenschaftlich viel Potenzial vorhanden, die Behandlungsoptionen für HPP weiter zu verbessern.

\section{Fazit für die Praxis}

- Hypophosphatasie ist eine erbliche, metabolische Multisystemerkrankung mit einem breiten klinischen Spektrum an Symptomen.

- Eine fehlende bis reduzierte Aktivität der alkalischen Phosphatase (AP) ist ursächlich für Mineralisationsstörungen von Knochen und Zähnen sowie Muskel- und Gelenkschmerzen.
- Die Diagnose der HPP lässt sich anhand klinischer Symptome und typischer laborchemischer Veränderungen (AP-Erniedrigung, PLPErhöhung) stellen.

- Die molekulargenetische Untersuchung des ALPL-Gens wird zur Bestätigung der Diagnose und differentialdiagnostischen Abgrenzung, zur Einschätzung von Wiederholungswahrscheinlichkeiten bei zukünftigen Schwangerschaften und zur pränatalen Diagnose bei familiär vorkommender schwerer HPP angeboten.

- Es besteht eine gewisse Geno-Phänotyp-Korrelation, jedoch ist die individuelle Vorhersage des Phänotyps anhand eines Genbefunds nicht möglich.

- Eine interdisziplinäre klinische Betreuung des Patienten mit Vertretern v. a. der Disziplinen Pädiatrie, Osteologie, Orthopädie/Unfallchirurgie, Neurologie, Psychologie und Zahnmedizin/Kieferorthopädie ist von zentraler Bedeutung.

- Eine Enzymersatztherapie ist nach sorgfältiger individueller Indikationsstellung bei schweren Fällen mit nachgewiesener Knochenmanifes- 
tation und Erkrankungsbeginn vor dem 18. Lebensjahr in Erwägung zu ziehen.

\section{Korrespondenzadresse}

Prof. Dr. med. Florian Barvencik

Institut für Osteologie und Biomechanik (IOBM), Universitätsklinikum Hamburg-

Eppendorf

Lottestraße 59, 22529 Hamburg, Deutschland

fbarvencik@uke.de

\section{Einhaltung ethischer Richtlinien}

Interessenkonflikt. N.M. Jandl, A. Volk und F. Barvencik geben an, dass kein Interessenkonflikt besteht.

Für diesen Beitrag wurden von den Autoren keine Studien an Menschen oder Tieren durchgeführt. Für die aufgeführten Studien gelten die jeweils dort angegebenen ethischen Richtlinien.

Open Access. Dieser Artikel wird unter der Creative Commons Namensnennung 4.0 International Lizenz (http://creativecommons.org/licenses/by/4.0/deed. de) veröffentlicht, welche die Nutzung, Vervielfältigung, Bearbeitung, Verbreitung und Wiedergabe in jeglichem Medium und Format erlaubt, sofern Sie den/die ursprünglichen Autor(en) und die Quelle ordnungsgemäßnennen, einen Link zur Creative Commons Lizenz beifügen und angeben, ob Änderungen vorgenommen wurden.

\section{Literatur}

1. Mornet E (2018) Hypophosphatasia. Metabolism $82: 142-155$

2. Rathbun JC (1948) Hypophosphatasia; a new developmental anomaly. Am J Dis Child 75(6):822-831

3. Whyte MP (2016) Hypophosphatasia-aetiology, nosology, pathogenesis, diagnosis and treatment. Nat Rev Endocrinol 12(4):233-246

4. Kishnani PS, Rockman-Greenberg C, Rauch F, Bhatti MT, Moseley S, Denker AE, Watsky E, Whyte MP (2019) Five-year efficacy and safety of asfotase alfa therapy for adults and adolescents with hypophosphatasia. Bone 121:149-162

5. Schmidt T, Mussawy H, Rolvien T, Hawellek T, Hubert J, Ruther W, Amling M, Barvencik F (2017) Clinical, radiographic and biochemical characteristics of adult hypophosphatasia. Osteoporos Int 28(9):2653-2662

6. Colazo JM, Hu JR, Dahir KM, Simmons JH (2019) Neurological symptoms in hypophosphatasia. Osteoporos Int 30(2):469-480

7. Whyte MP, Zhang F, Wenkert D, McAlister WH, Mack KE, Benigno MC, Coburn SP, Wagy S, Griffin DM, Ericson KL, Mumm S (2015) Hypophosphatasia: validation and expansion of the clinical nosology for children from 25 years experience with 173 pediatric patients. Bone 75:229-239

8. Nielson CM, Zmuda JM, Carlos AS, Wagoner WJ, Larson EA, Orwoll ES, Klein RF (2012) Rare coding variants in ALPL are associated with low serum alkaline phosphatase and low bone mineral density. JBone Miner Res 27(1):93-103

9. Whyte MP (1994) Hypophosphatasia and the role of alkaline phosphatase in skeletal mineralization. Endocr Rev 15(4):439-461

10. Hawrylak K, Stinson RA (1987) Tetrameric alkaline phosphatase from human liver is converted to dimers by phosphatidylinositol phospholipase $C$. FEBSLett 212(2):289-291

11. Young GP, Rose IS, Cropper S, Seetharam S, Alpers DH (1984) Hepatic clearance of rat plasma intestinal alkaline phosphatase. Am J Physiol 247(4):G419-26

12. Millan JL, Whyte MP (2016) Alkaline phosphatase and hypophosphatasia. Calcif Tissue Int 98(4):398-416

13. Khan AA, Josse R, Kannu P, Villeneuve J, Paul T, Van Uum S, Greenberg CR (2019) Hypophosphatasia: Canadian update on diagnosis and management Osteoporos Int 30(9):1713-1722. https://doi.org/ 10.1007/s00198-019-04921-y

14. ALPL gene mutations database (2019) The tissue nonspecific alkaline phosphatase gene mutations database. http://www.sesep.uvsq.fr/03_hypo_ mutations.php.Zugegriffen:03.12.2019

15. Taillandier A, Sallinen SL, Brun-Heath I, De Mazancourt P, Serre JL, Mornet E (2005) Childhood hypophosphatasia due to a de novo missense mutation in the tissue-nonspecific alkaline phosphatase gene. J Clin Endocrinol Metab 90(4):2436-2439

16. Watanabe A, Satoh S, Fujita A, Naing BT, Orimo H, Shimada T (2014) Perinatal hypophosphatasia caused by uniparental isodisomy. Bone 60:93-97

17. Hancarova $M$, Krepelova A, Puchmajerova $A$, Soucek O, Prchalova D, SumnikZ, SedlacekZ (2015) Hypophosphatasia due to uniparental disomy. Bone 81:765-766

18. Mornet $E$ (2015) Molecular genetics of hypophosphatasia and phenotype-genotype correlations. Subcell Biochem 76:25-43

19. Zurutuza L, Muller F, Gibrat JF, Taillandier A, SimonBouy B, Serre JL, Mornet E (1999) Correlations of genotype and phenotype in hypophosphatasia. Hum Mol Genet 8(6):1039-1046

20. Ikenoue $S$, Miyakoshi K, Ishii T, Sato $Y$, Otani T, Akiba $Y$, Kasuga $Y$, Ochiai D, Matsumoto T, Ichihashi Y, Matsuzaki Y, Tachikawa K, Michigami T, Nishimura G, Ikeda K, Hasegawa T, Tanaka M (2018) Discordant fetal phenotype of hypophosphatasia in two siblings. Am J Med Genet A 176(1):171-174

21. Stevenson DA, Carey JC, Coburn SP, Ericson KL, Byrne JL, Mumm S, Whyte MP (2008) Autosomal recessive hypophosphatasia manifesting in utero with long bone deformity but showing spontaneous postnatal improvement. J Clin Endocrinol Metab 93(9):3443-3448

22. Taketani T, Onigata K, Kobayashi H, Mushimoto $Y_{\text {, }}$ Fukuda S, Yamaguchi S (2014) Clinical and genetic aspects of hypophosphatasia in Japanese patients. Arch Dis Child 99(3):211-215

23. Mornet E, Yvard A, Taillandier A, Fauvert D, SimonBouy B (2011) A molecular-based estimation of the prevalence of hypophosphatasia in the European population. Ann Hum Genet 75(3):439-445

24. Wenkert D, McAlister WH, Coburn SP, Zerega JA, Ryan LM, Ericson KL, Hersh JH, Mumm S, Whyte MP (2011) Hypophosphatasia: nonlethal disease despite skeletal presentation in utero (17 new cases and literature review). J Bone Miner Res 26(10):2389-2398

25. Whyte MP, Wenkert D, McAlister WH, Mughal MZ Freemont AJ, Whitehouse R, Baildam EM, Coburn SP, Ryan LM, Mumm S (2009) Chronic recurrent multifocal osteomyelitis mimicked in childhood hypophosphatasia. J Bone Miner Res 24(8):1493-1505

26. Guanabens N, Mumm S, Moller I, Gonzalez-Roca E, Peris P, Demertzis JL, Whyte MP (2014) Calcific periarthritis as the only clinical manifestation of hypophosphatasia in middle-aged sisters. J Bone Miner Res 29(4):929-934

27. Chuck AJ, Pattrick MG, Hamilton E, Wilson R, Doherty M (1989) Crystal deposition in hypophosphatasia: a reappraisal. Ann Rheum Dis 48(7):571-576

28. Whyte MP, McAlister WH, Patton LS, Magill HL, Fallon MD, Lorentz WB Jr., Herrod HG (1984) Enzyme replacement therapy for infantile hypophosphatasia attempted by intravenous infusions of alkaline phosphatase-rich Paget plasma: results in three additional patients. J Pediatr 105(6):926-933

29. Schmidt T, Rolvien T, Linke C, Jandl NM, Oheim R, Amling M, BarvencikF (2019) Outcome of teriparatide treatment on fracture healing complications and symptomatic bone marrow edema in four adult patients with hypophosphatasia. JBMR Plus 3(8):e10215. https://doi.org/10.1002/jbm4.10215

30. Seefried L, Baumann J, Hemsley S, Hofmann C, Kunstmann $E$, Kiese $B$, Huang $Y$, Chivers $S$, Valentin MA, Borah B, Roubenoff $R$, Junker U, Jakob F (2017) Efficacy of anti-sclerostin monoclonal antibody BPS804 in adult patients with hypophosphatasia. JClin Invest 127(6):2148-2158

31. Whyte MP, Greenberg CR, Salman NJ, Bober MB, McAlister WH, Wenkert D, Van Sickle BJ, Simmons JH, Edgar TS, Bauer ML, Hamdan MA, Bishop N, Lutz RE, McGinn M, Craig S, Moore JN, Tay lor JW, Cleveland RH, CranleyWR, Lim R, ThacherTD Mayhew JE, Downs M, Millan JL, Skrinar AM, CrineP, Landy H (2012) Enzyme-replacement therapy in life-threatening hypophosphatasia. N Engl J Med 366(10):904-913

32. Europäische Arzneimittel-Agentur (2019) Strensiq Zusammenfassung der Merkmale des Arzneimittels. https://www.ema.europa.eu/en/documents/ product-information/strensiq-epar-productinformation de.pdf.Zugegriffen:03.12.2019

33. Whyte MP, Simmons JH, Moseley S, Fujita KP, Bishop N, Salman NJ, Taylor J, Phillips D, McGinn M, McAlister WH (2019) Asfotase alfa for infants and young children with hypophosphatasia: 7 year outcomes of a single-arm, open-label, phase 2 extension trial. Lancet Diabetes Endocrinol 7(2):93-105

34. Rolvien T, Schmidt T, Schmidt FN, von Kroge $S$, Busse B, Amling M, Barvencik F (2019) Recovery of bone mineralization and quality during asfotase alfa treatment in an adult patient with infantileonset hypophosphatasia. Bone 127:67-74

35. Klidaras P, Severt J, Aggers D, Payne J, Miller PD, Ing SW (2018) Fracture healing in two adult patients with hypophosphatasia after asfotase alfa therapy. JBMRPlus 2(5):304-307

36. Shapiro JR, Lewiecki EM (2017) Hypophosphatasia in adults: clinical assessment and treatment considerations. JBone Miner Res 32(10):1977-1980 
Hier steht eine Anzeige.

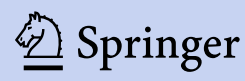

